\title{
Intermittent fasting: the science of going without
}

$\mathrm{M}$ any diet and exercise trends have origins in legitimate science, though the facts tend to get distorted by the time they achieve mainstream popularity. Benefits are exaggerated. Risks are downplayed. Science takes a back seat to marketing.

One needn't look any further than the emerging trend of intermittent fasting for a prime example. Advocates for taking periodic breaks from eating - for up to 24 hours once or twice a week - tout it as an effective and research-backed means of losing weight and improving health. That message has been reaching more and more ears of late.

"Right now, we are at a really important juncture for fasting," says Brad Pilon, an expert on intermittent fasting and author of the book Eat Stop Eat. "It's becoming extremely popular."

So popular, in fact, that it is quickly moving into fad territory, suggests Pilon. And when something becomes a fad - intensely popular but only for a short period - several problems typically ensue. For one, he says, many doctors and nutrition experts are prone to dismissing fads out of hand. So their patients and clients, while shielded from the ridiculous claims of overzealous dieting evangelists, may also lose out on the legitimate benefits of fasting done right. You know, the baby and bathwater thing.

Another concern is that promoters of intermittent fasting will, perhaps unintentionally, encourage extreme behaviour, such as bingeing. This is reflected in the photos accompanying many recent new articles on "the fast diet" or the " $5: 2$ diet." Often, they depict people eating heaps of highcalorie, high-fat foods, such as hamburgers, french fries and cake. The implication being that if you fast two days a week, you can devour as much junk as your gullet can swallow during the remaining five days.

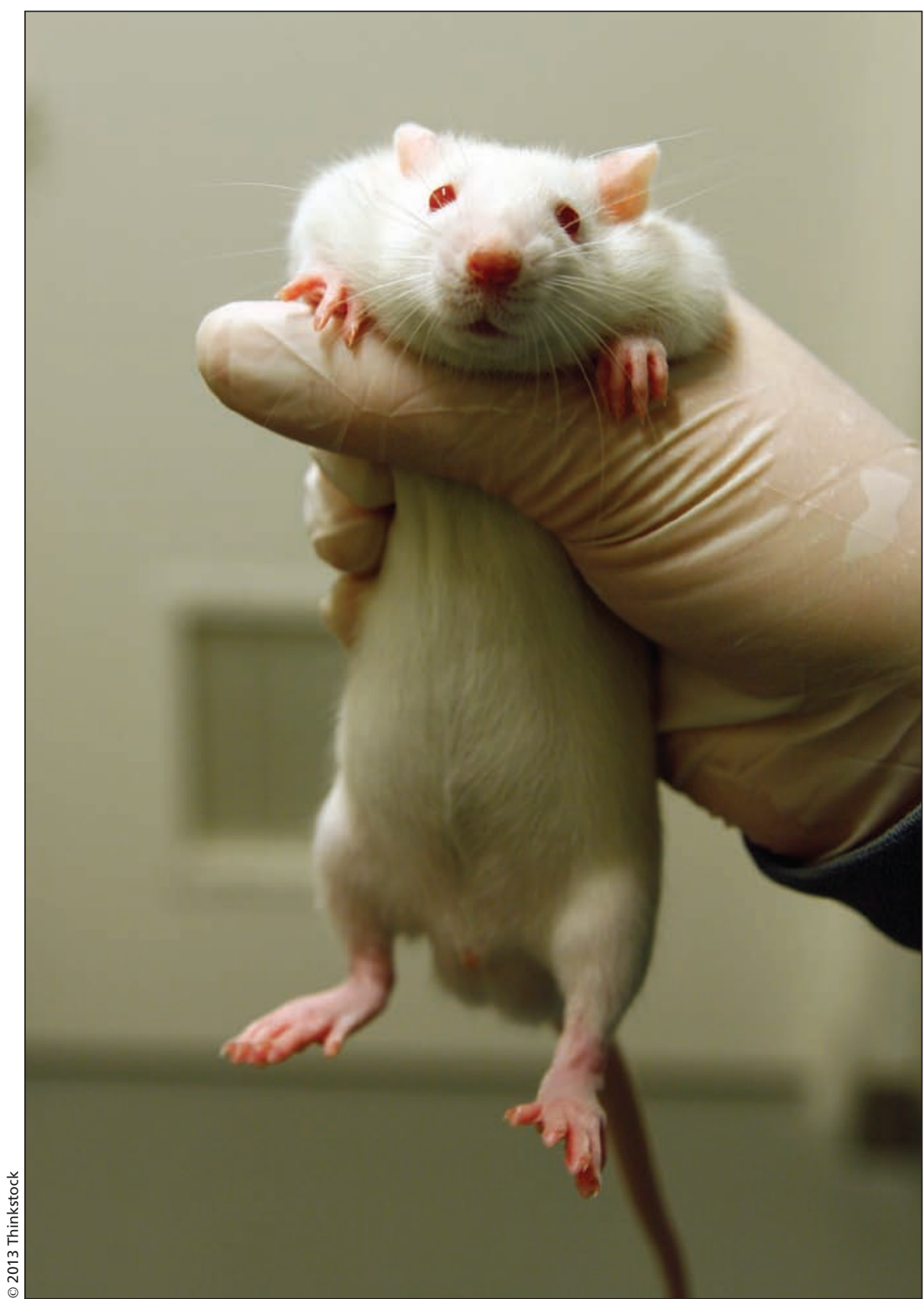

There is a large body of evidence that suggests fasting can benefit both the body and brain, but most research has been conducted on animals, such as mice. Researchers studying fasting are calling for more human studies.

Not so, say more moderate proponents of fasting. Their take on intermittent fasting: eat sensibly most of the time, eat nothing for an extended period every now and then, indulge only on occasion (perhaps once a week, say, on a designated "cheat day"). There is research, they claim, to back up the health benefits of sensibly incorporating fasting into your lifestyle.

There is indeed a large body of research to support the health benefits of fasting, though most of it has been conducted on animals, not humans. 
Still, the results have been promising. Fasting has been shown to improve biomarkers of disease, reduce oxidative stress and preserve learning and memory functioning, according to Mark Mattson, senior investigator for the National Institute on Aging, part of the US National Institutes of Health. Mattson has investigated the health benefits of intermittent fasting on the cardiovascular system and brain in rodents, and has called for "well-controlled human studies" in people "across a range of body mass indexes" ( $J$ Nutr Biochem 2005;16:129-37).

There are several theories about why fasting provides physiological benefits, says Mattson. "The one that we've studied a lot, and designed experiments to test, is the hypothesis that during the fasting period, cells are under a mild stress," he says. "And they respond to the stress adaptively by enhancing their ability to cope with stress and, maybe, to resist disease."

Though the word "stress" is often used in a negative sense, taxing the body and mind has benefits. Consider vigorous exercise, which stresses, in particular, muscles and the cardiovascular system. As long as you give your body time to recover, it will grow stronger. "There is considerable similarity between how cells respond to the stress of exercise and how cells respond to intermittent fasting," says Mattson.

Mattson has contributed to several other studies on intermittent fasting and caloric restriction. In one, overweight adults with moderate asthma consumed only $20 \%$ of their normal calorie intake on alternate days (Free Radical Bio Med 2007;42:665-74). Participants who adhered to the diet lost $8 \%$ of their initial body weight over eight weeks. They also saw a decrease in markers of oxidative stress and inflammation, and improvement of asthma-related symptoms and several quality-of-life indicators.

In another study, Mattson and colleagues explored the effects of intermittent and continuous energy restriction on weight loss and various biomarkers (for conditions including breast cancer, diabetes and cardiovascular disease) among young overweight woman (Int J Obesity 2011;35:714-27). They found that intermittent restriction was as effective as continuous restriction for improving weight loss, insulin sensitivity and other health biomarkers.

Mattson has also researched the protective benefits of fasting to neurons. If you don't eat for 10-16 hours, your body will go to its fat stores for energy, and fatty acids called ketones will be released into the bloodstream. This has been shown to protect memory and learning functionality, says Mattson, as well as slow disease processes in the brain.

But perhaps it isn't so much the fasting that produces health benefits, per se, as the resulting overall reduction in calorie intake (if, that is, you don't overeat on nonfasting days, which could create a caloric surplus instead of a deficit). That appears, at least, to be the case in slowing diseases such as cancer in mice, according to Dr. Stephen Freedland, associate professor of urology and pathology at the Duke University Medical Center in Durham, North Carolina.

"Caloric restriction, undernutrition without malnutrition, is the only experimental approach consistently shown to prolong survival in animal models," Freedland and colleagues stated in a study on the effects of intermittent fast- ing on prostate cancer growth in mice (Prostate Cancer Prostatic Dis 2010; 13:350-5). In the study, mice fasted twice a week for 24 hours, but were otherwise permitted to eat at liberty. During nonfasting days, the mice overate. Overall, they did not lose weight, counteracting whatever benefits they might have seen from fasting. Intermittent fasting with compensatory overeating "did not improve mouse survival nor did it delay prostrate tumor growth," the study concluded.

To improve health, the goal should be to lose weight by reducing the total amount of calories consumed, suggests Freedland, rather than focusing on when those calories are consumed. "If you [don't] eat two days a week, and limit what you eat the other five days, you will lose weight. It's one approach to losing weight," he says. "I'm not sure it works any better than cutting down slightly seven days a week."

People should also be wary of books written for broad audiences that explain the science behind fasting or any other health trend, he says. One purpose of writing a book for the consumer market, after all, is to sell as many copies as possible. Authors tend to present only evidence supporting their point of view, suggests Freedland, while ignoring evidence that contradicts it. "It's a lot of spin when you write a book." Roger Collier, CMAJ

CMAJ 2013. DOI:10.1503/cmaj.109-4451

Editor's note: This is a follow-up to a previous news story at cmaj.ca: "Intermittent fasting: the next big weight loss fad." 